\title{
The prognostic role of intragenic copy number breakpoints and identification of novel fusion genes in paediatric high grade glioma
}

\author{
Diana Carvalho ${ }^{1,2,3}$, Alan Mackay ${ }^{1}$, Lynn Bjerke ${ }^{1}$, Richard G Grundy ${ }^{4}$, Celeste Lopes ${ }^{2}$, Rui M Reis ${ }^{3,5}$ and Chris Jones ${ }^{1 *}$
}

\begin{abstract}
Background: Paediatric high grade glioma ( $\mathrm{pHGG)}$ is a distinct biological entity to histologically similar tumours arising in older adults, and has differing copy number profiles and driver genetic alterations. As functionally important intragenic copy number aberrations (iCNA) and fusion genes begin to be identified in adult HGG, the same has not yet been done in the childhood setting. We applied an iCNA algorithm to our previously published dataset of DNA copy number profiling in PHGG with a view to identify novel intragenic breakpoints.

Results: We report a series of 288 iCNA events in $\mathrm{PHGG}$, with the presence of intragenic breakpoints itself a negative prognostic factor. We identified an increased number of iCNA in older children compared to infants, and increased iCNA in H3F3A K27M mutant tumours compared to G34R/N and wild-type. We observed numerous gene disruptions by iCNA due to both deletions and amplifications, targeting known HGG-associated genes such as RB1 and NF1, putative tumour suppressors such as FAF1 and KIDINS220, and novel candidates such as PTPRE and KCND2. We further identified two novel fusion genes in PHGG - CSGALNACT2:RET and the complex fusion DHX57:TMEM178: MAP4K3. The latter was sequence-validated and appears to be an activating event in pHGG.
\end{abstract}

Conclusions: These data expand upon our understanding of the genomic events driving these tumours and represent novel targets for therapeutic intervention in these poor prognosis cancers of childhood.

Keywords: Fusion, Paediatric, Glioblastoma, Copy number, Intragenic

\section{Background}

DNA copy number and gene expression studies have highlighted key distinctions between high grade gliomas (HGG) arising in childhood and far more commonly, much later in adult life [1-4]. Indeed, recent exome-level sequencing initiatives have conclusively shown the existence of subgroups of HGG marked by distinct driver mutations [5], which are significantly enriched in young children (H3F3A K27M), teenagers and young adults (H3F3A G34R/V), and middle-aged adults $(I D H 1 / 2)$ [6]. Specific driving events for infants and elderly patients remain to be elucidated, however they too represent biological sub-entities, with infants having few genomic alterations [4], and elderly patients harbouring frequent amplification of EGFR and other genomic events [2,3].

\footnotetext{
* Correspondence: chris.jones@icr.ac.uk

'Divisions of Molecular Pathology and Cancer Therapeutics, Institute of Cancer Research, 15 Cotswold Road, Sutton, Surrey SM2 5NG, UK Full list of author information is available at the end of the article
}

The identification of driving genetic alterations at the DNA copy level are necessarily most commonly focussed on assessing the amplification/deletion of genes in their entirety, and approaches to ascribe significance to genomic events make use of overlapping regions across multiple samples to find genes consistently within regions of gain/loss [7]. This approach has the result of ignoring genes for whom the breakpoint, i.e. the specific location of copy number change, is found within the coding regions. Such events may be more than mere bystanders of the "driving" aberration, and may themselves play significant roles in tumour initiation and maintenance.

One key implication of copy number breakpoints occurring within genes is the possibility of generating novel fusions. Gene fusions can occur through both intra- and inter-chromosomal translocations, bringing together coding regions from two or more genes within a single reading frame allowing expression of a novel protein. Such gene fusions are common in cancer, but 
have historically been thought to be largely restricted to haematological malignancies and selected solid tumours such as sarcomas. Recent evidence has overturned this, with numerous novel gene fusions being discovered in a wide range of cancer types, exemplified by the identification of common TMPRSS2:ERG fusions in prostate cancer [8] and the EML4:ALK fusion in non-small cell lung cancer [9].

The first fusion gene found in glioblastoma was the rearrangement located at an amplified region at chromosome $4 \mathrm{q} 12$, resulting in the fusing of the kinase domain of PDGFRA with the regulatory domains of KDR (VEGFR2) [10]. This KDR:PDGFRA was found to be activating and tumorigenic, however to date only a single additional case has been found, in a paediatric high grade glioma (pHGG) [11], and thus these fusions do not represent a common event. Another low frequency fusion has more recently been identified in approximately 3\% of adult HGG, involving FGFR1 or FGFR3 partnering with TACC1 or TACC3 [12]. These FGFR:TACC fusions have been shown to localize to mitotic spindle poles, have constitutive kinase activity and induce mitotic and chromosomal segregation defects and aneuploidy [12]. The types of integrated analysis that identified these mutations have also begun to identify more common rearrangements, such as numerous fusions involving $E G F R$, the most frequently seen partner producing the EGFR-SEPT14 fusion demonstrated to activate STAT3 signaling and confer mitogen independence and sensitivity to EGFR inhibition [13].

Such analyses are clearly proving extremely valuable in furthering our understanding of HGG biology and generating novel targets for therapeutic intervention. As similar approaches are yet to be undertaken in the paediatric setting, we have applied an algorithm designed to identify intragenic copy number breakpoints in our previously published study of DNA copy number [4]. We identify numerous potentially functional gene disruptions and a novel validated complex fusion, DHX57:TMEM178: MAP4K3.

\section{Methods}

\section{Published DNA copy number data}

We previously carried out a DNA copy number profiling study of 100 pHGG cases on Affymetrix 500 K SNP arrays [4]. The data have been deposited at the Gene Expression Omnibus (GEO, www.ncbi.nlm.nih.gov/geo/) with accession number GSE19578. Copy number assignment was carried out as per the original publication, using Affymetrix Genotyping Analysis Software (GTYPE version 4.s) improved using Bayesian Linear Model with Mahalanobis distance classifier algorithm (BRLMM) and standard dChipSNP normalization and model-based expression algorithms [4]. $\log _{2}$-transformed data was used for all subsequent analysis in the present study.

\section{iCNA algorithm}

We implemented the iCNA algorithm developed as part of the GTS package under R2.11.0 (cbio.mskcc.org/ brennan) [14]. Breakpoints are calculated according to user-defined 'delta' values representing shifts in $\log _{2}$ ratios between two contiguous genomic regions after segmenting the copy number data using circular binary segmentation (cbs) [15]. Using a delta of 0.4 , breakpoint boundaries are identified and errors estimated by permutation-based calculations of neighbouring probe data. Confidence intervals are assigned and those falling within the $95 \%$ window considered 'high confidence'. An estimate is calculated for the expected rate of breaks for each gene based upon gene size and rate of breaks per sample, with a p value obtained based upon (observed-expected)/standard error. A corrected $\mathrm{p}$ value of $<0.05$ is considered significant. Manual inspection of copy number plots was undertaken to ensure sufficient probe coverage was present at identified loci in order to prioritise the most convincing breakpoints. Those with substantial gaps at either side of the break were excluded.

\section{Custom oligonucleotide array CGH}

We designed two fine-tiling oligonucleotide microarrays to cover the specific amplicons observed at chromosome 2p22.1 and 10q11.21 This was undertaken using the Agilent custom array design tool e-Array (Agilent, Santa Clara, CA, USA; https://earray.chem.agilent.com/), and comprised 700 probes covering $43.56-43.70 \mathrm{Mb}$ on chromosome 10 and 5000 probes covering $39-40 \mathrm{Mb}$ on chromosome 10 with a median probe interval of $200 \mathrm{bp}$ on $2 \times 105 \mathrm{~K}$ microarray. Due to limited amount of material, DNA was whole genome amplified (WGA) using the GenomePlex ${ }^{\circledast}$ Complete Whole Genome Amplification Kit (Sigma, Gillingham, UK) starting with $10 \mathrm{ng}$ of sample and control DNA, and following the manufacturer's protocol. WGA DNA was labelled using the Agilent Genomic DNA ULS labelling kit, hybridised as per manufacturer's instructions, and scanned on the Agilent 2505B Microarray Scanner System. Data has been submitted to ArrayExpress with accession number E-MTAB-2340.

\section{siRNA knockdown}

siRNA was carried out using a Dharmacon SMARTpool ${ }^{\mathrm{TM}}$ (Dharmacon, Lafayette, CO, USA) against MAP4K3 (\#003588) with paediatric glioma cells SF188, KNS42, UW479, Res259 and Res186 [16] and a panel of breast carcinoma lines. Cells were plated and transfected 24 hours later with siRNA using Lipofectamine RNAiMax ${ }^{\mathrm{TM}}$ (Invitrogen, Paisley, UK) as per manufacturer's instructions, alongside transfections of siControl. Twenty four hours following transfection, cells were trypsinised and media replenished after 48 hours and 96 hours, with cell viability assessed after seven days using CellTiter-Glo ${ }^{\mathrm{\tau \mu}}$ Luminescent Cell Viability 
Assay (Promega, Madison, WI, USA) as per manufacturer's instructions.

\section{Results}

Intragenic copy number breakpoints in paediatric HGG

We have previously carried out DNA copy number profiling of a large series of pHGG samples using Affymetrix $500 \mathrm{~K}$ SNP arrays, and reported numerous genes encompassed within areas of focal amplification and deletion [4]. We now applied an algorithm (iCNA [14]) designed to identify copy number breakpoints contained within the sequence of known genes. A full schema of the analytical process is given in Figure 1.
This algorithm was applied to 100 pHGG and 26 matched normal DNA samples, resulting in the identification of 1099 unique DNA copy number breaks contained within gene sequences across all tumour samples (Additional file 1: Table S1). Of these, 479 were found to map to known regions of copy number variations found commonly in the germlines of the general population by cross-referencing the breakpoints with The Centre for Applied Genomics Database of Genomic Variants [17], leaving a total of 620 events.

These were filtered to 500 after excluding those with $\mathrm{p}$ values $>0.05$, and further reduced to 388 with at least one sample harbouring a given aberration at high

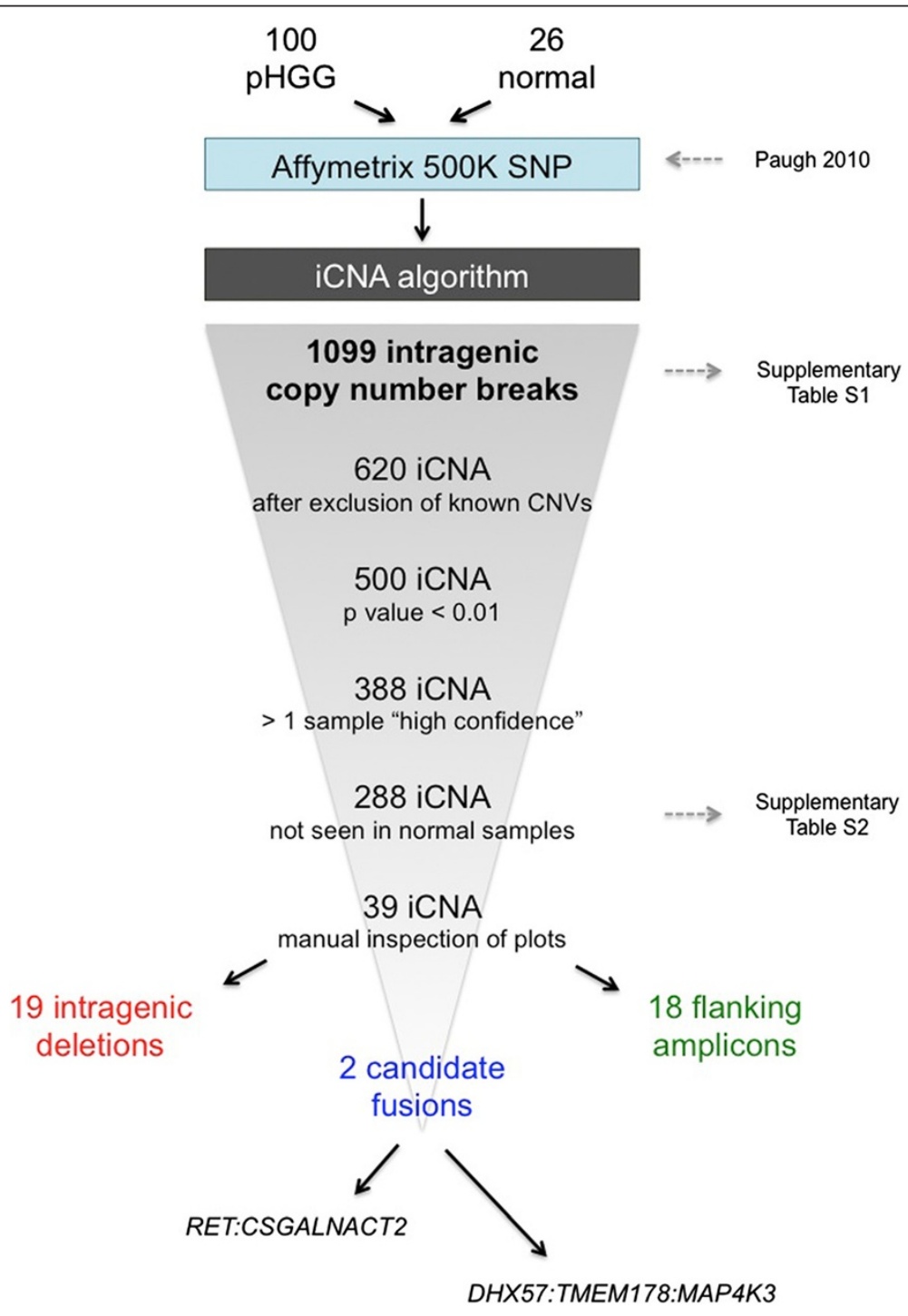

Figure 1 Schema of iCNA algorithm applied to paediatric high grade glioma. 1099 intragenic breakpoints were initially identified in a published series of $100 \mathrm{pHGG}$. From these, known copy number polymorphisms were excluded, as were these seen in a series of 26 matched normal DNA samples. After filtering for statistical significance and manual inspection of copy number plots, a series of 19 intragenic deletions, 18 amplicons, and 2 candidate fusions were identified. 
confidence'. A further 100 of these were excluded as they were also found in at least one of the normal samples profiled, representing either technical artifacts associated with the array platform used, or low frequency normal copy number polymorphisms. A final list of 288 iCNA is provided in Additional file 2: Table S2.

Most pHGG samples harboured at least one iCNA (median $=3$ ), although seven cases were found to contain none. Several cases were found to contain many more aberrations (maximum =19), though these were in the minority (Figure 2a). The number of iCNA events per sample was found to be prognostic in this multiinstitutional series of cases, with pHGG containing more than 10 iCNA $(n=9)$ found to have a significantly poorer survival (median $=7.8$ months), and those with no iCNA a better survival (median $=24$ months) than the rest of the tumours (median $=13.2$ months $)(p=0.026$, log-rank test $)$ (Figure 2b).

There were no differences in the number of iCNA between grade III $(\mathrm{n}=20$, median $=3$, range $0-7)$ and grade IV tumours $(\mathrm{n}=58$, median $=3$, range $0-19) \quad(\mathrm{p}=0.456$, $t$-test), though the cases with the highest number of iCNA were all grade IV glioblastoma (Figure 2c). Similarly, there were no differences between primary tumours $(n=68$, median $=3$, range 0-19) and those which arose as secondary malignancies after cranio-spinal irradiation ( $\mathrm{n}=10$, median $=2.5$, range $0-11)(\mathrm{p}=0.698, t$-test $)$ (Figure $2 \mathrm{~d})$. Infants (less than 3 years at diagnosis) had significantly fewer iCNA $(n=10$, median $=2.5$, range $0-6)$ than older children $(\mathrm{n}=68$, median $=4$, range $0-19) \quad(\mathrm{p}=0.050$, $t$-test) (Figure 2e). Tumours with the $\mathrm{K} 27 \mathrm{M}$ mutation in the gene encoding the histone variant H3.3, H3F3A, harboured significantly more iCNA $(\mathrm{n}=5$, median $=6$, range 1-16) than either G34R/V mutant tumours ( $n=4$, median $=4.5$, range $3-7)$ or wild-type $(n=14$, median $=3$, range $0-11)(p=0.043$, ANOVA) (Figure $2 \mathrm{f})$. This was independent of location of tumour, with no differences in number of iCNA between supratentorial GBM $(n=51)$ and DIPG ( $\mathrm{n}=7, \mathrm{p}=0.684, t$-test) (data not shown).

\section{Intragenic amplifications and deletions}

The 288 iCNA were further subjected to individual manual inspection of the data plots in order to identify the most robust copy number shifts associated with intragenic breaks. This resulted in a list of 39 unique events in 51 samples (Table 1). The recurrent changes included copy number loss, resulting primarily in either the absence of the 3' end of a gene or small deletions wholly within the coding sequence. These intragenic deletions included those targeting known tumour suppressors in glioblastoma
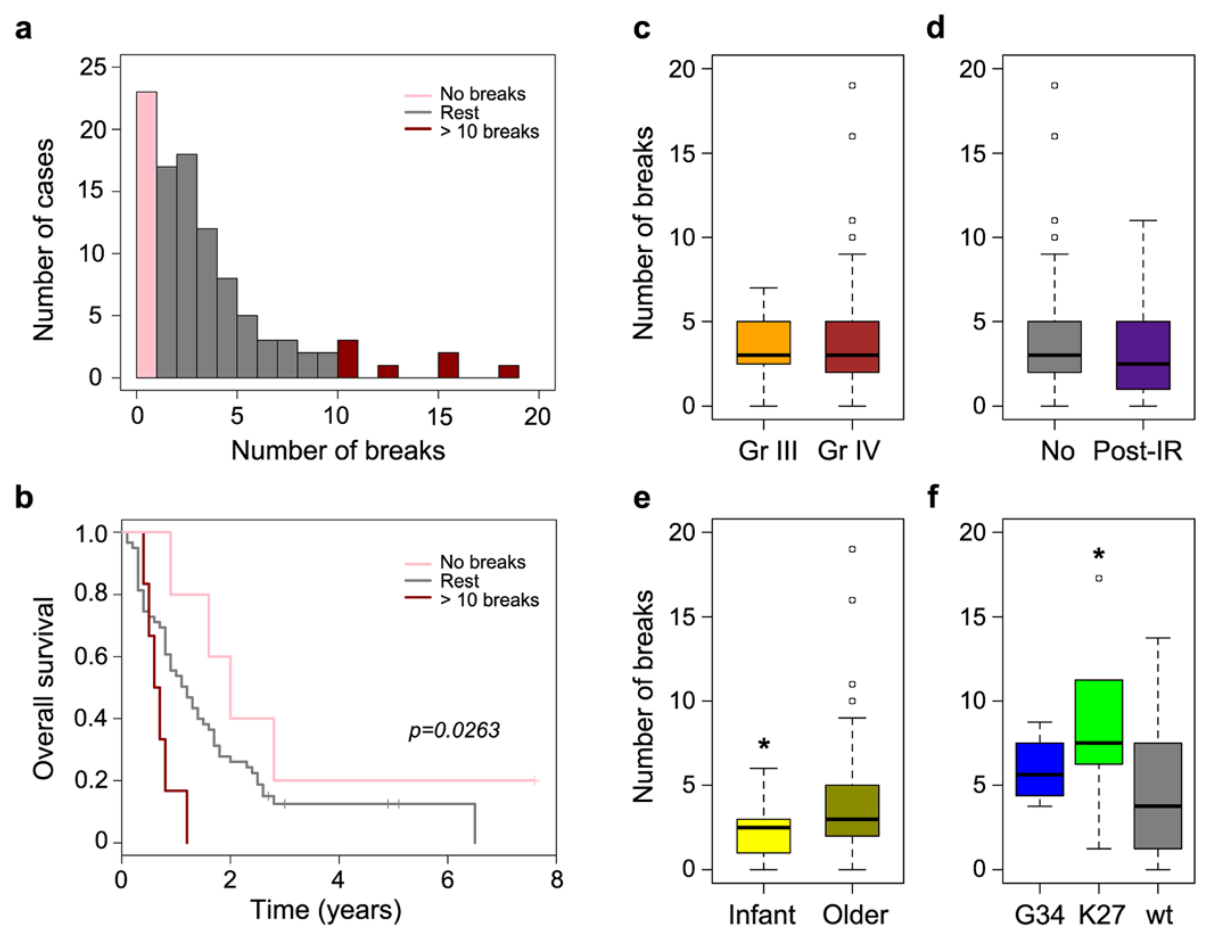

Figure 2 Clinicopathological correlates of intragenic copy number breaks in paediatric high grade glioma. (a) Number of intragenic breaks per sample in a series of 100 pHGG. (b) Kaplan-Meier plot of overall survival stratified by number of intragenic breaks per sample. Pink: no breaks; Red: more than 10 breaks per sample; Grey: rest. (c-f) Boxplot of number of intragenic breaks per sample separated by - (c) WHO grade. Orange: grade III; Brown: grade IV. (d) Previous radiation treatment for an earlier malignancy. Grey: primary pHGG; Purple: post-irradiation; (e) Age at diagnosis. Yellow: infant (<3 years old); Dark yellow: older children (>3 years old). (f) H3F3A status. Blue: G34R/V; Green: K27M; Grey: wild-type. 
Table 1 Nominated intragenic copy number aberration candidates

\begin{tabular}{|c|c|c|c|c|c|}
\hline Candidate.ID & Gene & Chromosome & Sample & Copy number change & Comments \\
\hline \multirow[t]{2}{*}{1} & FAF1 & 1 & HGG091 & Deletion & \\
\hline & FAF1 & 1 & HGG140 & Deletion & \\
\hline 2 & CD84 & 1 & HGG088 & Amplification & \\
\hline 3 & LGALS8 & 1 & HGG070 & Deletion & \\
\hline 4 & KIDINS220 & 2 & HGG077 & Amplification & \\
\hline 5 & DHX57 & 2 & HGG063 & Amplification & Fusion candidate \\
\hline 6 & TMEM178 & 2 & HGG063 & Amplification & Fusion candidate \\
\hline 7 & WDR49 & 3 & HGG010 & Amplification & \\
\hline 8 & PDCD10 & 3 & HGG157 & Deletion & \\
\hline 9 & $\mathrm{CHIC2}$ & 4 & HGG077 & Amplification & \\
\hline 10 & $|\mathrm{TGA}|$ & 5 & HGG029 & Deletion & \\
\hline 11 & EPHA7 & 6 & HGG139 & Deletion & \\
\hline 12 & LANCL2 & 7 & HGG060 & Amplification & \\
\hline 13 & ECOP & 7 & HGG060 & Amplification & \\
\hline \multirow[t]{2}{*}{14} & KCND2 & 7 & HGG152 & Amplification & \\
\hline & KCND2 & 7 & HGG162 & Amplification & \\
\hline 14 & SND1 & 7 & HGG090 & Deletion & \\
\hline \multirow[t]{3}{*}{15} & CSMD3 & 8 & HGG054 & Deletion & \\
\hline & CSMD3 & 8 & HGG140 & Deletion & \\
\hline & CSMD3 & 8 & HGG153 & Deletion & \\
\hline \multirow[t]{2}{*}{16} & SLC24A2 & 9 & HGG151 & Deletion & \\
\hline & SLC24A2 & 9 & HGG011 & Deletion & \\
\hline \multirow[t]{2}{*}{17} & MTAP & 9 & HGG022 & Deletion & \\
\hline & MTAP & 9 & HGG007 & Deletion & \\
\hline 18 & ANKRD26 & 10 & HGG068 & Deletion & \\
\hline 19 & RET & 10 & HGG139 & Amplification & Fusion candidate \\
\hline 20 & CSGALNACT2 & 10 & HGG139 & Amplification & Fusion candidate \\
\hline \multirow[t]{2}{*}{21} & PTPRE & 10 & HGG086 & Deletion & \\
\hline & PTPRE & 10 & HGG145 & Deletion & \\
\hline 22 & RAB6IP1 & 11 & HGG092 & Amplification & \\
\hline 23 & PSMA1 & 11 & HGG092 & Amplification & \\
\hline \multirow[t]{2}{*}{24} & TMTC1 & 12 & HGG010 & Amplification & \\
\hline & TMTC1 & 12 & HGG068 & Amplification & \\
\hline 25 & LRRK2 & 12 & HGG068 & Amplification & \\
\hline 26 & MYO1A & 12 & HGG029 & Amplification & \\
\hline 27 & XRCC6BP1 & 12 & HGG029 & Amplification & \\
\hline \multirow[t]{2}{*}{28} & OSBPL8 & 12 & HGG065 & Amplification & \\
\hline & OSBPL8 & 12 & HGG162 & Amplification & \\
\hline 29 & RB1 & 13 & HGG154 & Deletion & \\
\hline 30 & PCDH17 & 13 & HGG059 & Deletion & \\
\hline 31 & CD276 & 15 & HGG006 & Deletion & \\
\hline 32 & MEF2A & 15 & HGG011 & Deletion & \\
\hline 33 & DNAH2 & 17 & HGG143 & Amplification & \\
\hline
\end{tabular}


Table 1 Nominated intragenic copy number aberration candidates (Continued)

\begin{tabular}{lllll}
\hline 34 & NF1 & 17 & HGG154 & Deletion \\
35 & NF1 & 17 & HGG140 & Deletion \\
36 & BRIP1 & 17 & HGG077 & Deletion \\
37 & KCNB1 & 20 & HGG139 & Amplification \\
& SYN3 & 22 & HGG017 & Deletion \\
38 & SYN3 & 22 & HGG146 & Deletion \\
39 & TIMP3 & 22 & HGG146 & Deletion \\
\hline
\end{tabular}

39 unique intragenic breakpoints found within 51 cases of paediatric high grade glioma. Direction of copy number shift (gain/loss) is reported, as well as candidate fusion events.

such as NF1 (17q11.2, $\mathrm{n}=2)$ (Figure 3a) and RB1 (13q14.2, $\mathrm{n}=1$ ) (Additional file 3: Figure $\mathrm{S} 3$ ), as well as putative novel GBM-associated genes including FAF1 (1p33, n = 2) and MTAP (9p21.3, $\mathrm{n}=2$ ) (Additional file 3: Figure S3). In addition, there were novel deletions in the protein phosphatase PTPRE (10q26.2, $\mathrm{n}=2$ ) (Figure 3b) and recurrent internal microdeletions in the gene CSMD3 (CUB and Sushi multiple domains 3$)(8 q 23.3, n=3)$, all of which overlapped to result in the loss of exon 4 (Additional file 3: Figure S3).

Copy number gains within gene coding regions tended to be associated with regions flanking known oncogenic amplicons. These included amplification of the $M Y C N$ locus at chromosome 2p24.3, which in case HGG077 breaks within the coding region of the kinase D-interacting substrate KIDINS220 (Figure 4a); amplification of PDGFRA at 4q12, harbouring an iCNA in CHIC2 in the same case (though only covered by two probes); and recurrent breakpoints in the gene encoding the potassium voltage-gated channel KCND2 at 7q31.31 in association with amplification of $M E T$, though curiously this targeted either $5^{\prime}$ or $3^{\prime}$ ends in two different cases (Figure $4 \mathrm{~b}$ ). Similarly, common amplification events encompassing EGFR (7p12) and CDK4 (12q14) had intragenic breakpoints at both ends in cases HGG060 (LANCL2 and ECOP) and HGG029 (MYO1A and XRCC6BP1), respectively (Additional file 4: Figure S4).

\section{Identification of novel fusion genes}

For the most part, iCNA events resulted in an imbalance of certain regions of coding genes in isolation, with the predicted consequence a disruption of full-length gene expression. For certain events however, a $5^{\prime}$ end of one gene was found amplified at a similar copy number to a 3 ' end of a second gene within the same case. We reasoned that such instances may represent candidate fusion genes, and we identified two such examples in our cohort.

The first was at chromosome 10q11.21 and reflected a single amplicon, breaking within the genes RET and CSGALNACT2 such that we propose a hypothetical fusion gene encompassing the $5^{\prime}$ regulatory regions of
CSGALNACT2 and the $3^{\prime}$ kinase domain of RET. In order to determine the precise breakpoints to allow validation of this novel fusion, we designed custom oligonucleotide arrays spanning the amplicon in order to carry out high-resolution array $\mathrm{CGH}$ on the reference case HGG139, a relapse sample of glioblastoma in which this genomic event was not present in the primary tumour. Although the breakpoint for CSGALNACT2 was identified within intron 2, leaving the catalytic domains intact, the breakpoint within RET could not be accurately determined to closer than $10 \mathrm{~kb}$ between introns 1 and 2 (Additional file 5: Figure S5). As material was limited for this case, we were unable to confirm the precise nature of the putative CSGALNACT2:RET fusion by PCR-based techniques.

The second fusion candidate was located at an amplified region of chromosome 2p22.1 in case HGG063, an anaplastic astrocytoma. At Affymetrix $500 \mathrm{~K}$ SNP resolution, this appeared to be a single amplicon with breaks within the coding regions of the RNA helicase DHX57 and the transmembrane protein TMEM178 (Figure 5a). Applying the same approach as above, using custom-designed oligonucleotide arrays for high-resolution array CGH revealed two amplicons within this structure, with further intragenic breakpoints within the mitogen-activated protein kinase MAP4K3 (Figure 5b). Designing PCR primers to amplify across the highly specific breakpoints confirmed the presence of the fusion, which was further validated by direct sequencing (Figure 6).

The resultant fusion gene, DHX57:TMEM178:MAP4K3, is a complex three gene fusion formed from a series of intragenic breaks, amplifications and inversions to produce a sequence comprising exons 1-12 of DHX57, exons 2-4 of TMEM178 and exons 13-34 of MAP4K3, associated with regions of microhomology (Figure 6). This would produce a protein with the zinger finger and DEAD-like helicase domains of DHX57, the claudin family transmembrane domains of TMEM178 and the citron domain of MAP4K3. Selective knockdown of MAP4K3 by siRNA leads to a significant reduction in cell viability in five paediatric glioma cell lines as assayed by CellTiter Glo, an 


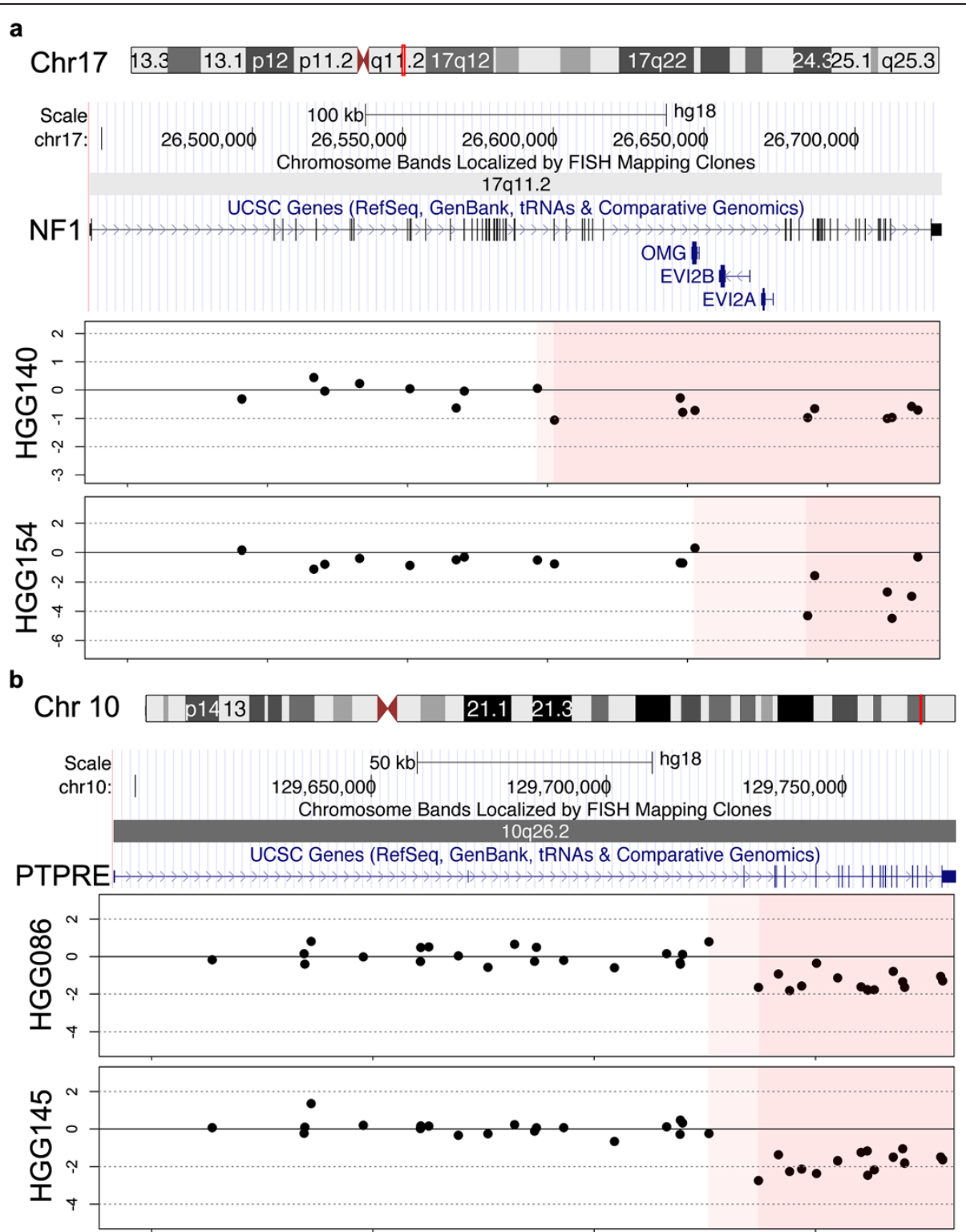

Figure 3 Intragenic deletions in paediatric high grade glioma. (a) Recurrent copy number breakpoint within NF1 on chromosome 17q11.2 in two cases of pHGG. (b) Recurrent copy number breakpoint within PTPRE on chromosome 10q26.2 in two cases of pHGG. Dark pink: confirmed region of loss; Light pink: region within which breakpoint lies, as defined by the resolution of probes on the array.

effect not seen in $18 / 20$ breast cancer cells $(p=0.0017$, pHGG $v s$ breast cancer, $t$-test) (Additional file 6: Figure S6).

\section{Discussion}

Comprehensive copy number profiling of adult and paediatric high grade gliomas was among the first data to demonstrate the biological differences between these similar-looking histological malignancies [18]. In this context, the focus has been on large-scale genomic copy number changes. A more refined analysis of copy number and exon-level expression data has identified new insights into genomic architecture and novel fusion proteins in adult glioblastoma [12,13]. Here we leverage a large dataset we have previously generated [4] in the paediatric disease to carry out a scan of intragenic breakpoints, leading to the identification of novel gene disruptions and candidate gene fusions.

The presence of intragenic copy number aberrations was confirmed in the vast majority of pHGG cases, and was itself prognostic, with an absence of iCNAs conferring a longer overall survival in paediatric patients. This was associated with the infant age group, known to have 
a

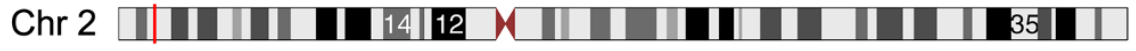

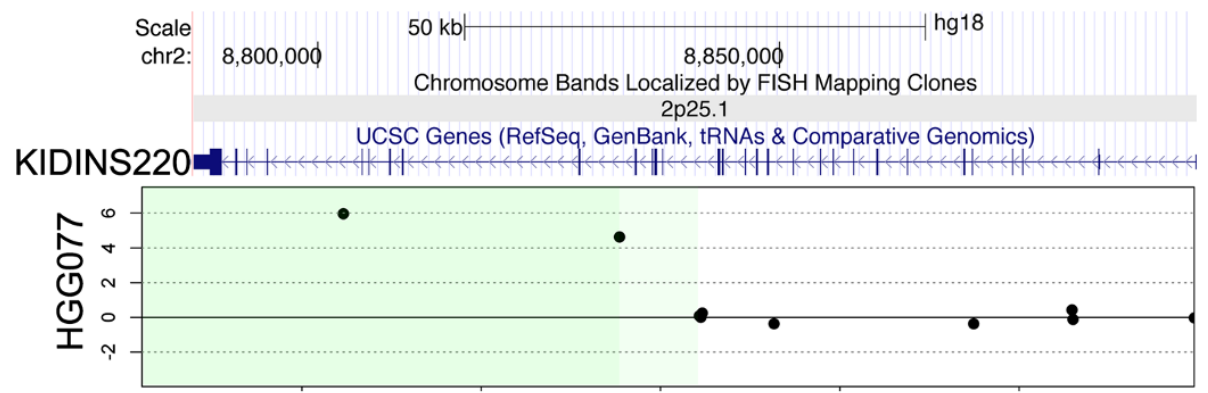

b

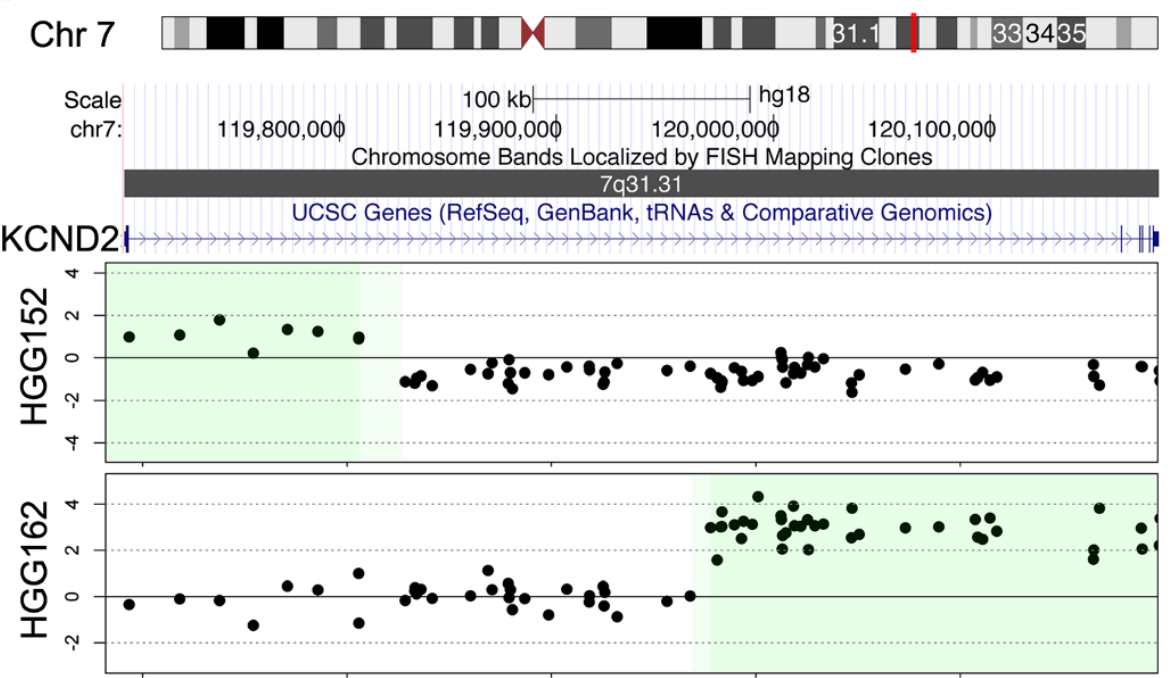

Figure 4 Intragenic amplifications in paediatric high grade glioma. (a) Copy number breakpoint within KIDINS200 on chromosome 2p25.1, flanking the MYCN amplicon in a case of pHGG. (b) Recurrent copy number breakpoint within KCND2 on chromosome $7 q 31.31$ in two cases of pHGG, in both cases part of the MET amplicon, though targeting either the 5' or $3^{\prime}$ end of the gene. Dark green: confirmed region of gain; Light green: region within which breakpoint lies, as defined by the resolution of probes on the array.

a better clinical outcome than older children [19], and further highlights the biological distinctiveness of this age group. By contrast, the presence of large numbers of intragenic breaks conferred a shorter survival time, but was not a result of the grade of the tumour, nor associated with a second malignancy due to radiation treatment for an earlier cancer. We had previously reported an association of post-irradiated HGG with PDGFRA amplification and chromosome 1q gain [4], so it appears these are relatively selective radiation-induced changes, rather than reflecting a generalised genomic instability in secondary tumours from these patients. Importantly, we identified an increased number of iCNA in tumours harbouring an $H 3 F 3 A \mathrm{~K} 27 \mathrm{M}$ mutation, regardless of anatomical location. This is a group of thalamic and pontine HGG associated with a particularly dismal prognosis [18], for whom understanding the mechanisms of genomic instability and the identification of novel gene disruptions is of considerable interest.

The majority of intragenic breakpoints we identified were associated with gene disruption. This includes deletions of known tumour suppressors such as $R B 1$ and $N F 1$, but also more novel glioblastoma associated genes. FAF1 and MTAP were both recurrently targeted by intragenic deletion events in pHGG. These genes are localised close to known cyclin-dependent kinase inhibitors and tumour suppressors $C D K N 1 C$ and $C D K N 2 A / B$, respectively, but both $F A F 1$ and MTAP have recently been proposed to harbour tumour suppressor activity in their own right. FAF1 is associated with a FAS-mediated apoptosis response and restoration of the FAF1 protein in adult glioma cell lines significantly increases cell death [20], whilst in MTAP-deficient cells, methylthioadenosine, generated during polyamine biosynthesis, is not 

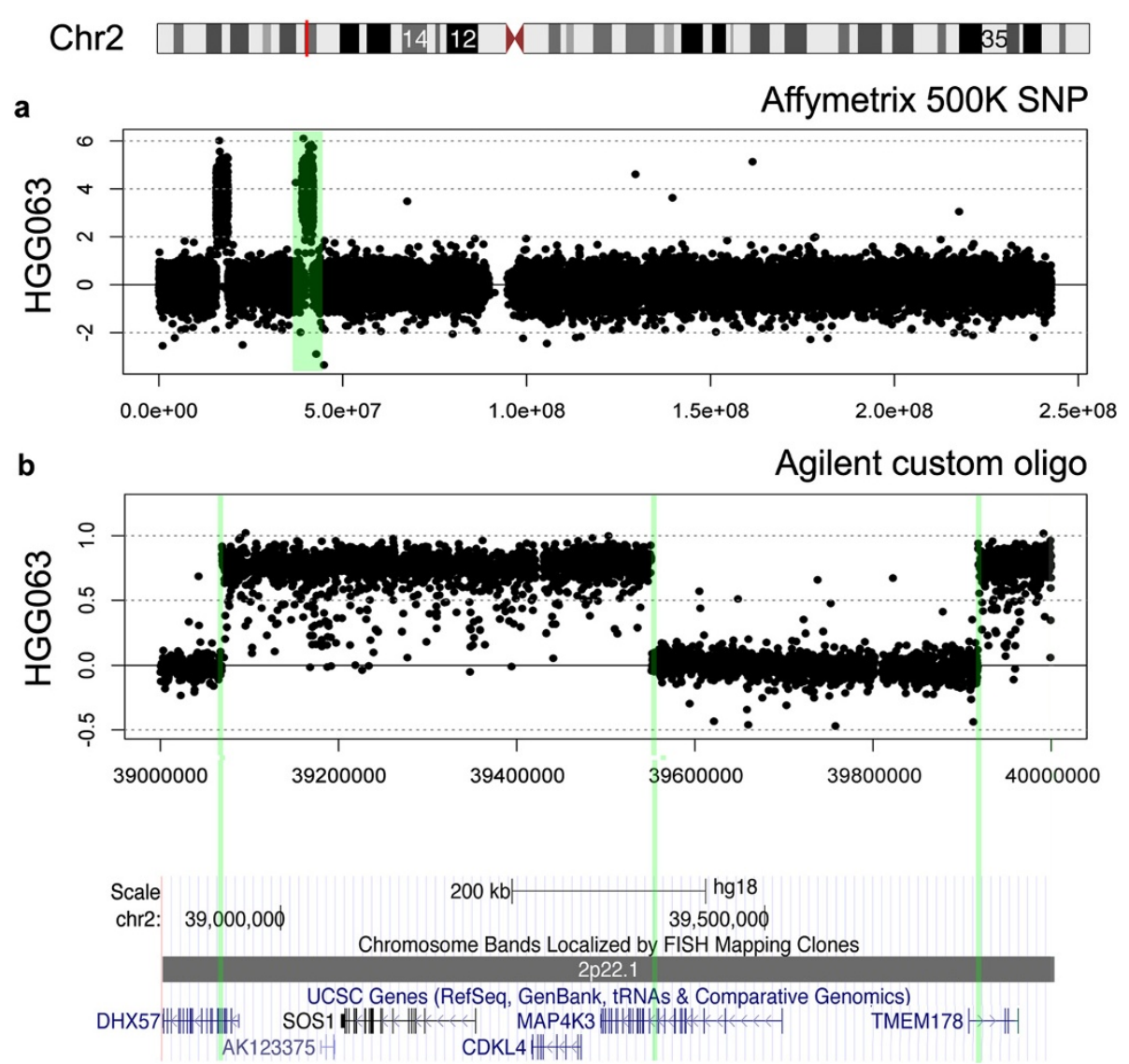

Figure 5 Identification of a novel complex fusion DHX57:TMEM178:MAP4K3. (a) Affymetrix 500 K SNP array of chromosome 2, highlighting two amplicons, the most telomeric encompassing MYCN, the more centromeric as 2p22.1 involving intragenic breakpoints in DHX57 and TMEM178 (green). (b) Custom oligonucleotide array of the 2p22.1 amplicon, revealing two amplified structures and three intragenic breakpoints, in DHX57, MAP4K3 and TMEM178 (green).

cleaved and the salvage pathway for adenine and methionine is absent [21]. It seems that such mechanisms are also likely in a subset of paediatric tumours.

Of note we identified novel deletions in the protein phosphatase epsilon, PTPRE. This has not been reported previously, although there are several reports of the tumour suppressive capacity of the related PTPRD $[22,23]$. This gene also appears targeted by intragenic deletions, and human astrocytes lacking PTPRD exhibited increased growth, as it is thought the protein usually functions to dephosphorylate the oncoprotein STAT3 [23]. The wholly intragenic microdeletions observed in CSMD3 in four cases may represent another novel mechanism of gene disruption. CSMD3 encodes a gene with multiple CUB and Sushi domains whose function is poorly understood. Recently, CSMD3 was identified as the second most frequently mutated gene (next to TP53) in lung cancer, where it was demonstrated that loss of CSMD3 results in increased proliferation of airway epithelial cells [24].
Gene disruption may also play a significant functional role when known gain-of-function oncogenes are amplified. We report numerous intragenic breakpoints which may have been overlooked in the context of identifying the 'driver' event within a common amplicon, but which may themselves be tumorigenic. These include disruptions of KIDINS220, a functional mediator of multiple receptor signalling pathways and essential for cortical development [25,26]; CHIC2, frequently deleted/rearranged in myeloid malignancies [27]; and KCND2, encoding a potassium voltage-gated channel, which is expressed in both neuronal and glial cells and has been shown to regulate ERK signalling in ganglioglioma [28]. All of these gene disruptions represent novel avenues for understanding the underlying biology of pHGG.

Of most interest was the use of the iCNA algorithm to identify potential novel fusion genes, as was demonstrated in adult glioblastoma with the identification of the KDR: PDGFRA fusion [10], which we also found in a case of pHGG [11]. Our analysis nominated two potential 


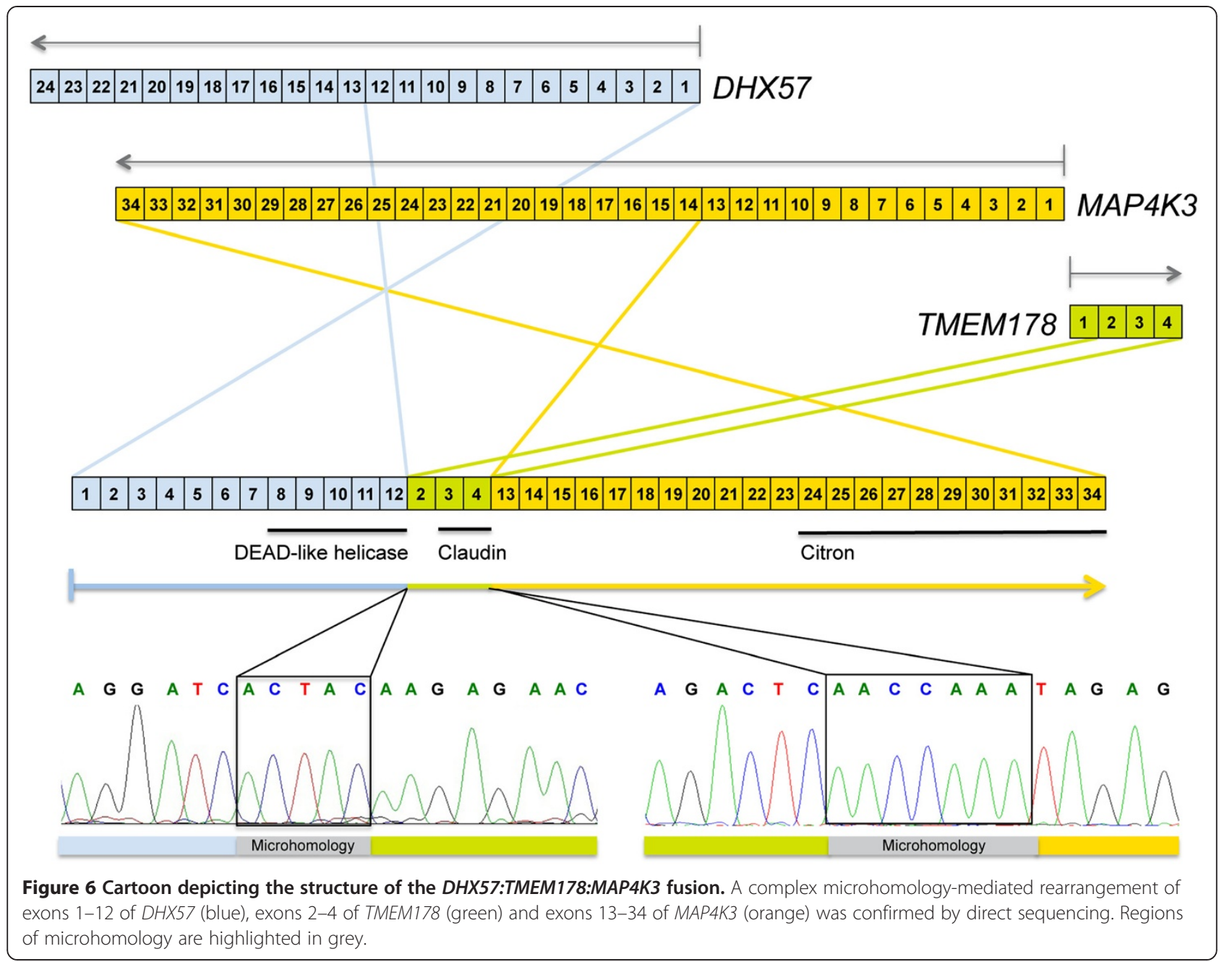

candidates - the first we were unable to conclusively validate, CSGALNACT2:RET. Such a putative fusion would retain the kinase domain of the RET oncoprotein, but would lose the autoregulatory portion of the protein, instead fusing it to the $\mathrm{N}$ terminal of chondroitin sulfate $\mathrm{N}$-acetyl-galactosaminyltransferase 2 . Although a precise cancer-related function has not been ascribed to the latter enzyme, it is though to play an important role in morphogenesis in zebrafish models [29,30]. Whilst not validated, oncogenic RET rearrangements and fusions are common in thyroid and lung cancer [31,32], and the presence of infrequent activating fusions in HGG do not seem unlikely.

We were able to validate a novel complex fusion involving three genes with intragenic breakpoints and amplification/rearrangement on chromosome $2 \mathrm{p} 22.1$. The resulting fusion gene, DHX57:TMEM178:MAP4K3 encompasses key regulatory domains from all three proteins, though a specific function is hard to predict. The helicase properties of the DHX57 component may be a candidate for oncogenicity, with numerous other DEAD-box helicases appearing to play a role in regulation of DNA repair, apoptosis and drug sensitivity [33]. MAP4K3 has been associated with several malignancies in both an oncogenic and tumour suppressor capacity [34,35]. In particular, one function that has been ascribed includes activation of mTOR signalling via the TORC1 complex [36], a pathway commonly activated by diverse mechanisms in pHGG [18]. In the context of pHGG, although the kinase domain is not retained in the fusion, MAP4K3 plays some functional role as selective knockdown by siRNA leads to a significant and selective reduction in cell viability in paediatric glioma cell lines. Thus we hypothesise that the DHX57:TMEM178:MAP4K3 is activating as disruption of the protein would otherwise seem incompatible with tumour cell growth and proliferation.

\section{Conclusion}

In summary these data represent a key addition to our understanding of the genomic alterations driving pHGG and provide novel avenues for developing sorely-needed novel therapeutic strategies for children with these otherwise incurable tumours. 


\section{Additional files}

Additional file 1: Table S1. Initial output from iCNA algorithm detailing 1099 intragenic copy number breakpoints in 100 cases of paediatric high grade glioma.

Additional file 2: Table S2. Final output from iCNA algorithm detailing 288 filtered intragenic copy number breakpoints in 100 cases of paediatric high grade glioma.

Additional file 3: Figure S3. Intragenic deletions in paediatric high grade glioma. (a) Recurrent copy number breakpoint within FAF1 on chromosome 1 p33 in two cases of pHGG. (b) Recurrent copy number breakpoint within MTAP on chromosome 9p21.3 in two cases of pHGG. (c) Copy number breakpoint within RB1 on chromosome 13q14.2 in a case of pHGG. (b) Recurrent copy number breakpoint within CSMD3 on chromosome 8q23.3 in three cases of pHGG. Dark pink: confirmed region of loss; Light pink: region within which breakpoint lies, as defined by the resolution of probes on the array.

Additional file 4: Figure S4. Intragenic amplifications in paediatric high grade glioma. (a) Copy number breakpoints within LANCL2 and ECOP on chromosome 7p11.2, flanking the EGFR amplicon in a case of pHGG. (b) Recurrent copy number breakpoints within MYO1A1 and XRCC6BP1 on chromosome 12q13.3 and 12q14.1, flanking the CDK4 amplicon in a case of pHGG. Dark green: confirmed region of gain; Light green: region within which breakpoint lies, as defined by the resolution of probes on the array.

Additional file 5: Figure S5. Identification of a novel candidate fusion CSGALNACT2:RET. (a) Affymetrix $500 \mathrm{~K} \mathrm{SNP}$ array of chromosome 10, highlighting an amplicon at 10q11.21 (green). (b) Custom oligonucleotide array of the 10q11.21 amplicon, revealing a clear breakpoint within CSGALNACT2 (green), but a less clear boundary within RET (grey).

Additional file 6: Figure S6. siRNA knockdown of MAP4K3 in paediatric glioma and breast carcinoma cells. Paediatric glioma cells (green) were highly sensitive to knockdown of MAP4K3, with $5 / 5$ cells showing significant effects on cell viability. By contrast, only $2 / 20$ breast cancer cells (blue) showed a similar dependency on MAP4K3 expression for cell viability. The screen was carried out in three independent experiments and was highly reproducible for all cell lines, with $R^{2}$ values ranging from 0.68-0.94 (breast) and 0.78-0.92 (glioma). The different sensitivity of glioma cells to MAP4K3 knockdown as compared to breast carcinoma cells was statistically significant ( $p=0.0017, \mathrm{pHGG}$ vs breast cancer, $t$-test).

\section{Competing interests}

The authors declare that they have no competing interests.

\section{Authors' contributions}

DC designed and performed experiments and analysed and interpreted data. LB designed and interpreted experiments. AM analysed and interpreted data. RG provided clinical samples. RMR, CL and CJ designed the study. DC and CJ wrote the manuscript. All authors read and approved the final manuscript.

\section{Acknowledgements}

We are grateful for support from the Rosetrees Trust, the Brain Tumour Charity and Fundação para a Ciência e Tecnologia, Portugal (PhD Studentship SFRH/BD/33473/2008). DC, AM, LB and CJ acknowledge NHS funding to the Biomedical Research Centre.

\section{Author details}

'Divisions of Molecular Pathology and Cancer Therapeutics, Institute of Cancer Research, 15 Cotswold Road, Sutton, Surrey SM2 5NG, UK. ${ }^{2}$ University of Coimbra, Palácio dos Grilos, R. da Ilha, Coimbra 3000-214, Portugal. ${ }^{3}$ Life and Health Sciences Research Institute (ICVS), School of Health Sciences, University of Minho, Braga, and ICVS/3B's-PT Government Associate Laboratory, Braga/Guimarães, Portugal. ${ }^{4}$ Childhood Brain Tumour Research Centre, University of Nottingham, Kings Meadow Campus, Lenton Lane, Nottingham NG7 2NR, UK. ${ }^{5}$ Molecular Oncology Research Center, Barretos Cancer Hospital, Barretos, SP, Brazil.
Received: 16 December 2013 Accepted: 11 February 2014

Published: 18 February 2014

\section{References}

1. Bax DA, Mackay A, Little SE, Carvalho D, Viana-Pereira M, Tamber N, Grigoriadis A, Ashworth A, Reis RM, Ellison DW, Al-Sarraj S, Hargrave D, Jones C: A distinct spectrum of copy number aberrations in paediatric high grade gliomas. Clin Cancer Res 2010, 16(13):3368-3377.

2. McLendon R, Friedman A, Bigner D, Van Meir EG, Brat DJ, Mastrogianakis M, Olson JJ, Mikkelsen T, Lehman N, Aldape K, Alfred Yung WK, Bogler O, Vandenberg S, Berger M, Prados M, Muzny D, Morgan M, Scherer S, Sabo A, Nazareth L, Lewis L, Hall O, Zhu Y, Ren Y, Alvi O, Yao J, Hawes A, Jhangiani S, Fowler G, San Lucas A, et al: Comprehensive genomic characterization defines human glioblastoma genes and core pathways. Nature 2008, 455:1061-1068.

3. Parsons DW, Jones $S$, Zhang $X$, Lin JC, Leary RJ, Angenendt $P$, Mankoo $P$, Carter H, Siu IM, Gallia GL, Olivi A, McLendon R, Rasheed BA, Keir S, Nikolskaya T, Nikolsky Y, Busam DA, Tekleab H, Diaz LA Jr, Hartigan J, Smith DR, Strausberg RL, Marie SK, Shinjo SM, Yan H, Riggins GJ, Bigner DD, Karchin R, Papadopoulos N, Parmigiani G, et al: An integrated genomic analysis of human glioblastoma multiforme. Science 2008, 321(5897):1807-1812.

4. Paugh BS, Qu C, Jones C, Liu Z, Adamowicz-Brice M, Zhang J, Bax DA, Coyle B, Barrow J, Hargrave D, Lowe J, Gajjar A, Zhao W, Broniscer A, Ellison DW, Grundy RG, Baker SJ: Integrated molecular genetic profiling of pediatric high-grade gliomas reveals key differences with the adult disease. J Clin Oncol 2010, 28(18):3061-3068.

5. Schwartzentruber J, Korshunov A, Liu XY, Jones DT, Pfaff E, Jacob K, Sturm D, Fontebasso AM, Quang DA, Tonjes M, Hovestadt V, Albrecht S, Kool M, Nantel A, Konermann C, Lindroth A, Jager N, Rausch T, Ryzhova M, Korbel JO, Hielscher T, Hauser P, Garami M, Klekner A, Bognar L, Ebinger M, Schuhmann MU, Scheurlen W, Pekrun A, Fruhwald MC, et al: Driver mutations in histone H3.3 and chromatin remodelling genes in paediatric glioblastoma. Nature 2012, 482(7384):226-231.

6. Sturm D, Witt H, Hovestadt V, Khuong-Quang DA, Jones DT, Konermann C, Pfaff E, Tonjes M, Sill M, Bender S, Kool M, Zapatka M, Becker N, Zucknick M, Hielscher T, Liu XY, Fontebasso AM, Ryzhova M, Albrecht S, Jacob K, Wolter M Ebinger M, Schuhmann MU, van Meter T, Fruhwald MC, Hauch H, Pekrun A, Radlwimmer B, Niehues T, von Komorowski G, et al: Hotspot mutations in $\mathrm{H} 3 \mathrm{~F} 3 \mathrm{~A}$ and IDH1 define distinct epigenetic and biological subgroups of glioblastoma. Cancer Cell 2012, 22(4):425-437.

7. Beroukhim R, Getz G, Nghiemphu L, Barretina J, Hsueh T, Linhart D, Vivanco I, Lee JC, Huang JH, Alexander S, Du J, Kau T, Thomas RK, Shah K, Soto H, Perner S, Prensner J, Debiasi RM, Demichelis F, Hatton C, Rubin MA, Garraway LA, Nelson SF, Liau L, Mischel PS, Cloughesy TF, Meyerson M, Golub TA, Lander ES, Mellinghoff IK, Sellers WR: Assessing the significance of chromosomal aberrations in cancer: methodology and application to glioma. Proc Natl Acad Sci USA 2007, 104(50):20007-20012.

8. Tomlins SA, Rhodes DR, Perner S, Dhanasekaran SM, Mehra R, Sun XW, Varambally S, Cao X, Tchinda J, Kuefer R, Lee C, Montie JE, Shah RB, Pienta KJ, Rubin MA, Chinnaiyan AM: Recurrent fusion of TMPRSS2 and ETS transcription factor genes in prostate cancer. Science 2005, 310(5748):644-648.

9. Soda M, Choi YL, Enomoto M, Takada S, Yamashita Y, Ishikawa S, Fujiwara S, Watanabe H, Kurashina K, Hatanaka H, Bando M, Ohno S, Ishikawa Y, Aburatani H, Niki T, Sohara Y, Sugiyama Y, Mano H: Identification of the transforming EML4-ALK fusion gene in non-small-cell lung cancer. Nature 2007, 448(7153):561-566.

10. Ozawa T, Brennan CW, Wang L, Squatrito M, Sasayama T, Nakada M, Huse JT, Pedraza A, Utsuki S, Yasui Y, Tandon A, Fomchenko El, Oka H, Levine RL, Fujii K, Ladanyi M, Holland EC: PDGFRA gene rearrangements are frequent genetic events in PDGFRA-amplified glioblastomas. Genes Dev 2010, 24(19):2205-2218.

11. Paugh BS, Zhu X, Qu C, Endersby R, Diaz AK, Zhang J, Bax DA, Carvalho D, Reis RM, Onar-Thomas A, Broniscer A, Wetmore C, Zhang J, Jones C, Ellison DW, Baker SJ: Novel Oncogenic PDGFRA Mutations in Pediatric High-Grade Gliomas. Cancer Res 2013, 73(20):6219-6229.

12. Singh D, Chan JM, Zoppoli P, Niola F, Sullivan R, Castano A, Liu EM, Reichel J, Porrati P, Pellegatta S, Qiu K, Gao Z, Ceccarelli M, Riccardi R, Brat DJ, Guha A, Aldape K, Golfinos JG, Zagzag D, Mikkelsen T, Finocchiaro G, Lasorella A, 
Rabadan R, lavarone A: Transforming fusions of FGFR and TACC genes in human glioblastoma. Science 2012, 337(6099):1231-1235.

13. Frattini V, Trifonov V, Chan JM, Castano A, Lia M, Abate F, Keir ST, Ji AX, Zoppoli P, Niola F, Danussi C, Dolgalev I, Porrati P, Pellegatta S, Heguy A, Gupta G, Pisapia DJ, Canoll P, Bruce JN, McLendon RE, Yan H, Aldape K, Finocchiaro G, Mikkelsen T, Prive GG, Bigner DD, Lasorella A, Rabadan R, lavarone A: The integrated landscape of driver genomic alterations in glioblastoma. Nat Genet 2013, 45(10):1141-1149.

14. Wiedemeyer R, Brennan C, Heffernan TP, Xiao Y, Mahoney J, Protopopov A, Zheng H, Bignell G, Furnari F, Cavenee WK, Hahn WC, Ichimura K, Collins VP, Chu GC, Stratton MR, Ligon KL, Futreal PA, Chin L: Feedback circuit among INK4 tumor suppressors constrains human glioblastoma development. Cancer Cell 2008, 13(4):355-364.

15. Olshen $A B$, Venkatraman $E S$, Lucito R, Wigler M: Circular binary segmentation for the analysis of array-based DNA copy number data. Biostatistics 2004, 5(4):557-572.

16. Bax DA, Little SE, Gaspar N, Perryman L, Marshall L, Viana-Pereira M, Jones T, Williams RD, Vassal GM, Workman P, Sheer D, Reis RM, Pearson ADJ, Hargrave D, Jones C: Molecular and phenotypic characterisation of paediatric glioma cell lines as models for preclinical drug development. PLOS ONE 2009, 4(4):e5209.

17. lafrate AJ, Feuk L, Rivera MN, Listewnik ML, Donahoe PK, Qi Y, Scherer SW, Lee C: Detection of large-scale variation in the human genome. Nat Genet 2004, 36(9):949-951.

18. Jones C, Perryman L, Hargrave D: Paediatric and adult malignant glioma: close relatives or distant cousins? Nat Rev Clin Oncol 2012, 9(7):400-413.

19. Wu W, Lamborn KR, Buckner JC, Novotny PJ, Chang SM, O'Fallon JR, Jaeckle KA, Prados MD: Joint NCCTG and NABTC prognostic factors analysis for high-grade recurrent glioma. Neuro Oncol 2010, 12(2):164-172.

20. Zheng S, Fu J, Vegesna R, Mao Y, Heathcock LE, Torres-Garcia W, Ezhilarasan R, Wang S, McKenna A, Chin L, Brennan CW, Yung WK, Weinstein JN, Aldape KD, Sulman EP, Chen K, Koul D, Verhaak RG: A survey of intragenic breakpoints in glioblastoma identifies a distinct subset associated with poor survival. Genes Dev 2013, 27(13):1462-1472

21. Bertino JR, Waud WR, Parker WB, Lubin M: Targeting tumors that lack methylthioadenosine phosphorylase (MTAP) activity: current strategies. Cancer Biol Ther 2011, 11(7):627-632.

22. Solomon DA, Kim JS, Cronin JC, Sibenaller Z, Ryken T, Rosenberg SA, Ressom H, Jean W, Bigner D, Yan H, Samuels Y, Waldman T: Mutational inactivation of PTPRD in glioblastoma multiforme and malignant melanoma. Cancer Res 2008, 68(24):10300-10306.

23. Veeriah $S$, Brennan $C$, Meng S, Singh B, Fagin JA, Solit DB, Paty PB, Rohle D, Vivanco I, Chmielecki J, Pao W, Ladanyi M, Gerald WL, Liau L, Cloughesy TC, Mischel PS, Sander C, Taylor B, Schultz N, Major J, Heguy A, Fang F, Mellinghoff IK, Chan TA: The tyrosine phosphatase PTPRD is a tumor suppressor that is frequently inactivated and mutated in glioblastoma and other human cancers. Proc Natl Acad Sci USA 2009, 106(23):9435-9440.

24. Liu P, Morrison C, Wang L, Xiong D, Vedell P, Cui P, Hua X, Ding F, Lu Y, James M, Ebben JD, Xu H, Adjei AA, Head K, Andrae JW, Tschannen MR, Jacob H, Pan J, Zhang Q, Van den Bergh F, Xiao H, Lo KC, Patel J, Richmond T, Watt MA, Albert T, Selzer R, Anderson M, Wang J, Wang Y, Starnes S, Yang P, You M: Identification of somatic mutations in non-small cell lung carcinomas using whole-exome sequencing. Carcinogenesis 2012, 33(7):1270-1276.

25. Cesca F, Yabe A, Spencer-Dene B, Arrigoni A, Al-Qatari M, Henderson D, Phillips H, Koltzenburg M, Benfenati F, Schiavo G: Kidins220/ARMS is an essential modulator of cardiovascular and nervous system development. Cell Death Dis 2011, 2:e226.

26. Neubrand VE, Cesca F, Benfenati F, Schiavo G: Kidins220/ARMS as a functional mediator of multiple receptor signalling pathways. J Cell Sci 2012, 125(Pt 8):1845-1854.

27. Cools J, Bilhou-Nabera C, Wlodarska I, Cabrol C, Talmant P, Bernard P, Hagemeijer A, Marynen P: Fusion of a novel gene, BTL, to ETV6 in acute myeloid leukemias with a t (4;12) (q11-q12;p13). Blood 1999, 94(5):1820-1824

28. Adams JP, Anderson AE, Varga AW, Dineley KT, Cook RG, Pfaffinger PJ, Sweatt JD: The A-type potassium channel Kv4.2 is a substrate for the mitogen-activated protein kinase ERK. J Neurochem 2000, 75(6):2277-2287.

29. Filipek-Gorniok B, Holmborn K, Haitina T, Habicher J, Oliveira MB, Hellgren C, Eriksson I, Kjellen L, Kreuger J, Ledin J: Expression of chondroitin/dermatan sulfate glycosyltransferases during early zebrafish development. Dev Dyn 2013, 242(8):964-975.

30. Holmborn K, Habicher J, Kasza Z, Eriksson AS, Filipek-Gorniok B, Gopal S, Couchman JR, Ahlberg PE, Wiweger M, Spillmann D, Kreuger J, Ledin J: On the roles and regulation of chondroitin sulfate and heparan sulfate in zebrafish pharyngeal cartilage morphogenesis. J Biol Chem 2012, 287(40):33905-33916.

31. Fusco A, Santoro M: 20 years of RET/PTC in thyroid cancer: clinicopathological correlations. Ara Bras Endocrinol Metabol 2007, 51(5):731-735.

32. Gainor JF, Shaw AT: Novel targets in non-small cell lung cancer: ROS1 and RET fusions. Oncologist 2013, 18(7):865-875.

33. Fuller-Pace FV: DEAD box RNA helicase functions in cancer. RNA Biol 2013, 10(1):121-132

34. Lam D, Dickens D, Reid EB, Loh SH, Moisoi N, Martins LM: MAP4K3 modulates cell death via the post-transcriptional regulation of $\mathrm{BH} 3$-only proteins. Proc Natl Acad Sci USA 2009, 106(29):11978-11983.

35. Wright JH, Wang X, Manning G, LaMere BJ, Le P, Zhu S, Khatry D, Flanagan PM, Buckley SD, Whyte DB, Howlett AR, Bischoff JR, Lipson KE, Jallal B: The STE20 kinase HGK is broadly expressed in human tumor cells and can modulate cellular transformation, invasion, and adhesion. Mol Cell Biol 2003, 23(6):2068-2082

36. Findlay GM, Yan L, Procter J, Mieulet V, Lamb RF: A MAP4 kinase related to Ste20 is a nutrient-sensitive regulator of mTOR signalling. Biochem J 2007, 403(1):13-20.

doi:10.1186/2051-5960-2-23

Cite this article as: Carvalho et al:: The prognostic role of intragenic copy number breakpoints and identification of novel fusion genes in paediatric high grade glioma. Acta Neuropathologica Communications 2014 2:23.

\section{Submit your next manuscript to BioMed Central and take full advantage of:}

- Convenient online submission

- Thorough peer review

- No space constraints or color figure charges

- Immediate publication on acceptance

- Inclusion in PubMed, CAS, Scopus and Google Scholar

- Research which is freely available for redistribution
() Biomed Central 